\title{
VISOR DEL RÉGIMEN JURÍDICO DEL TERRITORIO: HERRAMIENTA PARA LA TOMA DE DECISIONES EN LA GOBERNANZA DEL TERRITORIO
}

\author{
Rafael Martínez Cebolla \\ Instituto Geográfico de Aragón, \\ Edificio Pignatelli, Paseo María Agustín, 36, 50004 Zaragoza, España \\ rmartinezceb@aragon.es \\ David Portolés Rodríguez \\ dportoles@idearium.eu \\ Fernando López Martín \\ Instituto Geográfico de Aragón, \\ Edificio Pignatelli, Paseo María Agustín 36, 50004 Zaragoza, España \\ flopezm@ragon.es
}

\begin{abstract}
Resumen: Todo plan, proyecto o iniciativa pública o privada que se realiza sobre el territorio ha de cumplir un marco legal. Este marco se modifica de diferentes formas a lo largo del tiempo. Y varía en función de la normativa que legisla el uso o aprovechamiento ya sea urbanístico, territorial, ambiental o sectorial. La realidad es que los actores públicos y privados quedan desbordados ante las relaciones jurídicas que entran en juego a la hora de realizar una determinada acción sobre el territorio. La herramienta del Régimen Jurídico del Territorio del Visor 2D de IDEARAGON es la respuesta del IGEAR para ayudar a los tomadores de decisiones dando cumplida respuesta de las limitaciones legales de diferente tipo en Aragón (España). La presente comunicación explica el por qué de la herramienta como ayuda a la gobernanza del territorio. Detalla el estado actual de la herramienta, así como, su evolución a corto plazo mediante la consulta diaria de los boletines oficiales.
\end{abstract}

Palabras clave: Lugar, Gobernanza, Legislación, Aragón, IDE.

Recibido: 04-06-2020. Aceptado: 08-07-2020. 


\begin{abstract}
Any public or private plan, project carried out on place must comply with a legal framework. This frame is modified in different ways over time. Depending on the regulations that legislate the use whether urban or territorial planning, environmental or thematic varies it. The reality is that public and private actors are exceeded by the legal relationships that come into play when taking a certain action on spatial location. IGEAR response with IDEARAGON 2D Viewer's Legal Regime of territory tool to help decision makers respond to legal limitations of different types in Aragon (Spain). This communication explains why the tool helps governance the territory. It details the tool as well as its short-term evolution through the daily query of official newsletters.
\end{abstract}

Keywords: Place, Governance, Legislation, Aragón, SDI.

\title{
1. Introducción
}

La Constitución de 1978 garantiza en su artículo 9.3 la publicidad de las normas. Es un imperativo legal canalizado a través de los distintos boletines oficiales. El Boletín Oficial del Estado (BOE), de acuerdo con el Real Decreto 181/2008, es el medio oficial para la publicación de leyes, reales decretos, decretos y disposiciones que recogen el paraguas legal del ordenamiento jurídico del territorio nacional. Contiene además las leyes aprobadas por las Cortes Generales así como las disposiciones generales de las Comunidades Autónomas. Y, por tanto, desde la aprobación de los sucesivos estatutos de autonomía se han afianzado las normativas autonómicas respectivas con la consecución de los diferentes boletines oficiales; el Boletín Oficial de Aragón (BOA) en el caso aragonés.

Esta publicidad detalla los derechos, así como los deberes y obligaciones de cualquier persona física o jurídica que vive o desarrolla una determinada actividad en el Estado Español. Esta garantía que se obtiene es una libertad más del ciudadano, pero confiere también una ardua y compleja tarea, vinculada a la proliferación de las normas jurídicas (de derecho administrativo, procesal, penal, etc.) si se atiende a su evolución histórica en las últimas décadas. Solo en datos cuantitativos, los boletines oficiales de España publicaron 2.615 páginas cada día de media en 2018, según informe de la CEOE (2019). Los boletines autonómicos publicitaron aproximadamente más de 790.000 páginas. Un gran volumen de las cuales, no forma parte del aparato normativo, pero sí una gran parte de publicidad, afecta a los intereses que puede tener cualquier actor público o privado en un determinado lugar.

La CEOE, desde el punto estrictamente económico, argumenta que "el marco normativo español se caracteriza por una elevada densidad y complejidad, lo que origina 
distorsiones en el mercado, y redunda en un alto nivel de obstáculos burocráticos y cargas administrativas para los operadores". Incide que "el marco normativo llega a ser desproporcionado y discriminatorio para los intereses de las personas físicas o jurídicas que quieren emprender una determinada actividad económica”.

"Profesionales legales son contratados para consultar regulaciones", pero "cuando la situación se convierte en prohibitiva financieramente, muchos ciudadanos y pequeñas empresas buscan ayuda a partir de intermediaros" (Sneiders, 2004). La realidad jurídico-normativa se convierte, por tanto, en un terreno vasto de diferente tipología que no es fácil de asimilar por parte de la sociedad. Requiere de conocimiento y tiempo para ser conocedor de todas las implicaciones que tiene el simple hecho del cumplimiento del deber u obligación. En el caso de Aragón, la evolución normativa refleja en los últimos seis años una producción de 109 normas con rango de Ley.

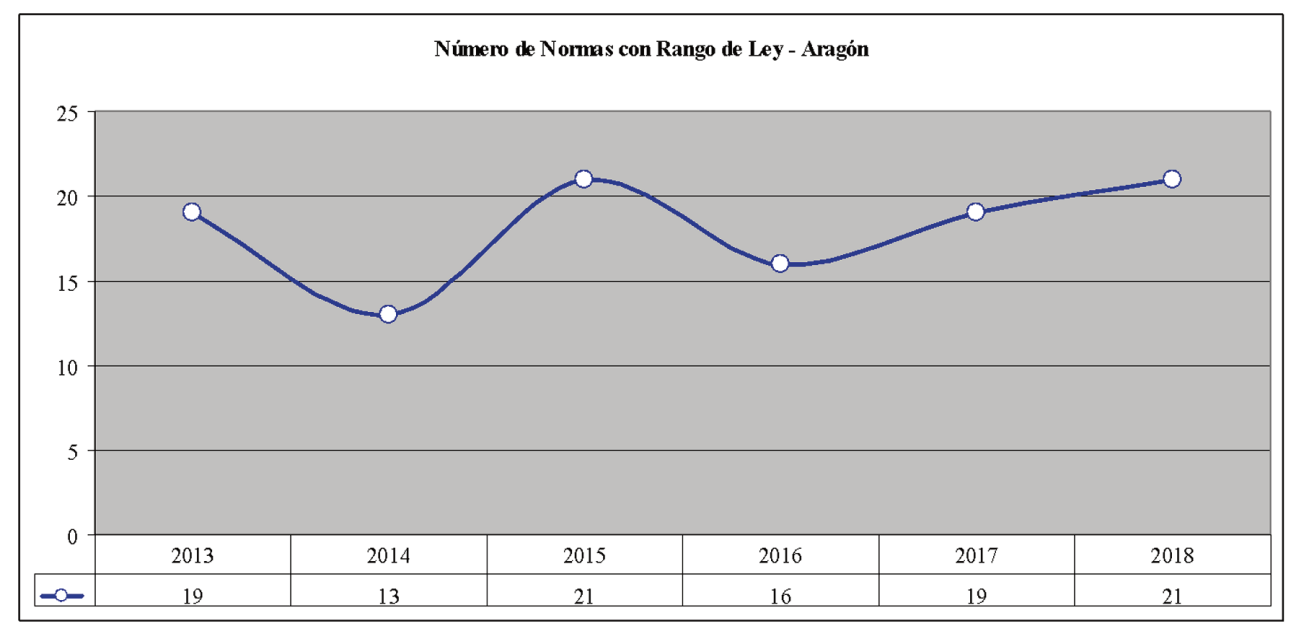

Figura 1. Evolución del Número de Normas con Rango de Ley en Aragón. Fuente: CEOE. Gráfico: Elaboración propia.

Solo 2018 emitió 44.204 páginas de información con una tasa de variación del 60,2\% desde el primer año de estudio de la serie en el año 2011. El número de actos legislativos así como acuerdos, sentencias y autos que se han realizado justifican la complejidad del marco legal vigente.

Cada hecho legal ocurre en un momento y ubicación concretos. Y dependiendo del evento que lo haya desencadenado, hay varias circunstancias procesales que deben ser consideradas. "La legislación siempre tiene un componente espacial, mediado a través del concepto de jurisdicción. Pero dependiendo del dominio de la ley, como la plani- 
ficación u ordenación del territorio, el espacio conceptual juega un papel clave" (Boer et al., 2007). Desde el punto de vista de la ordenación del territorio, el régimen jurídico del territorio es un agente pasivo para los agentes públicos y privados que quieren desarrollar una acción sobre el territorio. La ampliación de desarrollo urbano en un municipio, la construcción de un parque eólico o cualquier otra infraestructura desencadena un impacto que puede ser analizado desde varios puntos de vista (ambiental, económico, sociocultural). Desde el punto de vista jurídico, se construye un marco legal teniendo en cuenta todos estos puntos de vista con el fin de establecer las condiciones para la ejecución de todas las acciones posibles permitidas o no permitidas.

La herramienta del Régimen Jurídico del Territorio (RJT) del Visor 2D de la Infraestructura de Datos Espaciales de Aragón (IDEARAGON), es la respuesta del Instituto Geográfico de Aragón (IGEAR) para ayudar a los tomadores de decisiones, dando cumplida respuesta de las limitaciones legales de diferente tipo existentes en territorio aragonés. Esta herramienta informa de las relaciones espaciales y jurídicas existentes. El RJT está diseñado para conseguir contestar todas las restricciones que existen sobre el territorio aragonés. Su objetivo es proporcionar una imagen completa sobre el impacto de todas las regulaciones relacionadas en un determinado lugar. El RJT es una solución tecnológica basada en el paradigma IDE. Permite cruzar el prisma legal con la realidad geográfica con la misión de informar a cualquier actor público o privado.

\section{Metodología}

\subsection{Infraestructura de Datos Espaciales}

La información geográfica se ha incrementado exponencialmente en la última década vinculada al desarrollo de las Tecnologías de la Información Geográfica (TIG) así como al paradigma del Big Data. A la cantidad de datos -volumen-, la multiplicidad de formatos y fuentes -variedad-, y el constante movimiento - variabilidad- que inicialmente se asocia a este paradigma, se ha sumado el valor -utilidad del dato-, la velocidad -por la cual el dato es generado-, la viscosidad -pluralidad del dato-, la visualización -tiene sentido porque sirve para la toma de decisiones- y la veracidad -calidadde la información. El IGEAR entendió que la forma de gestionar la información geográfica del territorio pasaba necesariamente por aplicar el paradigma IDE para asumir un control eficiente. De esta manera, el descubrimiento, la visualización, la descarga así como el encadenamiento de servicios geográficos le convertían en una plataforma transversal de información (geográfica) para la acción del Gobierno.

IDEARAGON es el resultado. Una plataforma de información geográfica "encargada de aunar y converger el componente político, geográfico, tecnológico y social" (Abad et al., 2012) que una IDE ha de tener. Como IDE regional surte de información geográfica y accede a los servicios geográficos interoperables proporcionados a otra escala ya sean 
competencial o estrictamente de trabajo. La metodología empleada es doble para representar mediante mapas la praxis jurídica que se desarrolla en el espacio. Por un lado, es analítico mediante el procesado diario automático de los boletines oficiales, mediante una herramienta exploratoria del IGEAR llamada GeoBOA y, por otro lado, es deductivo mediante la lectura de las referencias legales a las que hacen referencia los anuncios públicos. La primera, sirve para el expurgo y filtrado de las referencias catastrales, municipales o comarcales ${ }^{1}$ citadas en los boletines. Y, la segunda, clasifica la normativa y la almacena en la base de datos de IDEARAGON para su posterior explotación a través de la herramienta del RJT.

Este análisis operacional prepara las referencias geográficas directas e indirectas que tienen relación con el anuncio legal. Las referencias legales se almacenan en la base de datos geográfica ordenadas por temas y grupos de información. Esta clasificación se relaciona con las capas cartográficas y la normativa asociadas al territorio.

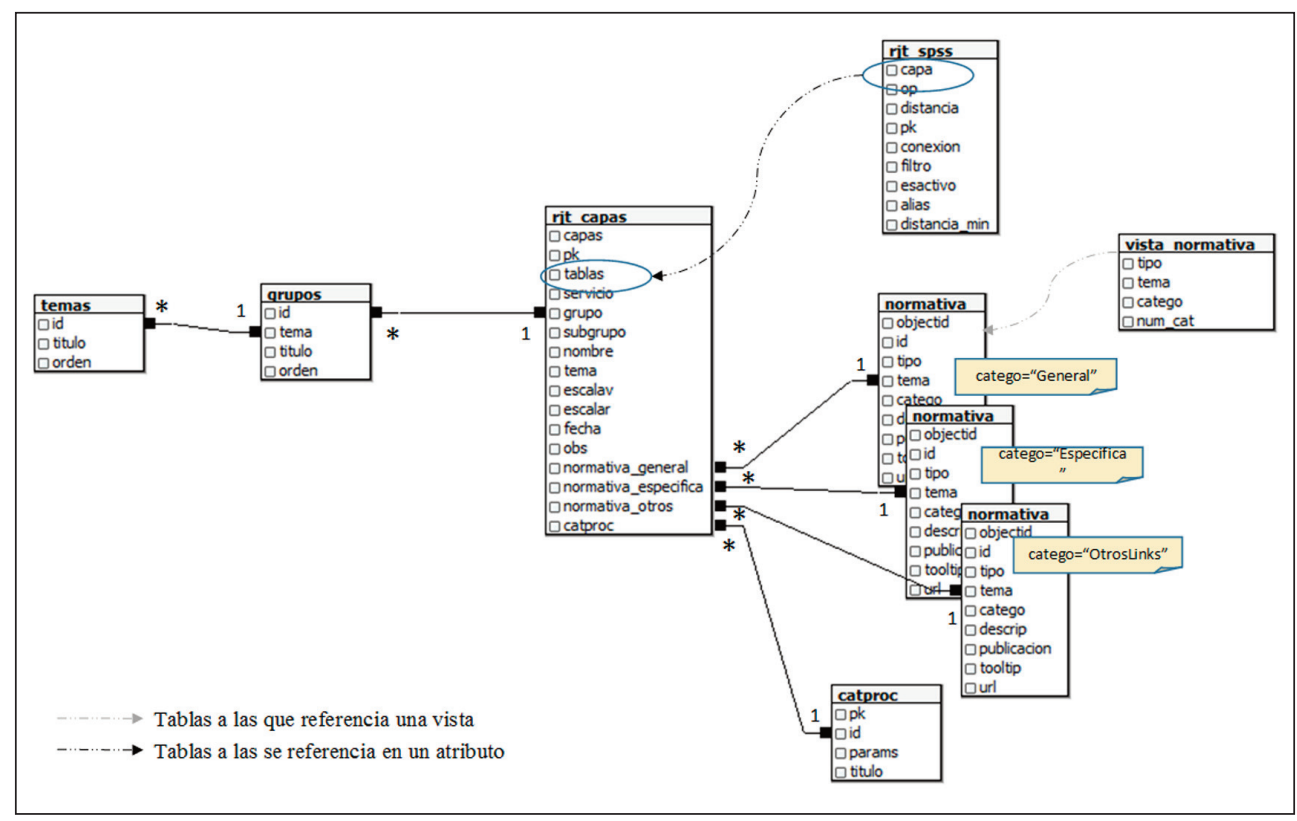

Figura 2. Modelo de datos lógico y físico de la herramienta RJT del Visor 2D.

Fuente: Elaboración propia.

1. El filtrado de referencias geográficas directas e indirectas se realizan también para coordenadas geográficas, nombres e identificadores de lugares protegidos de la Red Natura 2000 así como matrículas de terrenos cinegéticos y montes. 
Ambos tipos de información se clasifican en función del tipo de normativa y las limitaciones que aplican. La normativa aplicable a un determinado territorio es general, cuando la reglamentación establecida se ejecuta para todos los ámbitos geográficos determinados en la normativa. Es específica, cuando se desarrolla más regulación para un determinado espacio de la normativa general previamente establecida. La cartografía se representa asociada a la normativa con la mayor calidad y precisión posible. Aquel marco legal que lleva asociado más de una regulación, asociado generalmente a distancias, cruces con más información geográfica o ambas, se calcula dinámicamente a partir del dato geográfico original.

El despliegue operacional de carácter físico y lógico se realiza mediante la implementación de un modelo de datos de Entidad-Atributo-Relación. Este modelo implementa la herramienta del RJT generando una cascada de información geográfica y alfanumérica relacionada, que permite la consulta de las disposiciones legales que hay en un determinado espacio. En suma, este modelo es consultado a través de la arquitectura cliente-servidor de IDEARAGON y la aplicación web Visor 2D de IDEARAGON informa las limitaciones legales existentes en el territorio mediante la herramienta RJT.

\subsection{Fuentes de información}

La herramienta del RJT divide la información en tres grupos: Ordenación del Territorio y Urbanismo, ambiental y sectorial (incluye las restricciones relacionadas con redes de transporte, energía, minas y sector agroganadero). Cada grupo se organiza según el tema o competencia relacionada estableciendo una relación entre las fuentes de información y la regulación legal. Esta última, se obtiene directamente de los diarios oficiales y la información geográfica se obtiene de los departamentos y organismos autónomos del Gobierno de Aragón competentes por razón de la materia.

La relación anterior es un hecho clave del sistema porque requiere una acción humana. "Las normas se presentan en tres variantes principales en el lenguaje natural: obligaciones, permisos y prohibiciones. El uso de verbos no es indicativo de si algo es una norma o no" (Winkels et al., 2002). Por lo tanto, "un ser humano debe establecer claramente el mapeo entre los geodatos disponibles y el contenido de las normas descritas en el lenguaje natural con interpretaciones que serían semánticas ambiguas" (Portolés et al., 2019). Todos los datos geográficos son proporcionados por IDEARAGON, y su calidad es heterogénea, dependiendo de la escala y sus propios procesos de actualización. Después de seleccionar un grupo y tema, el usuario selecciona un ámbito geográfico para consultar sobre el régimen legal.

Después de esta consulta, el usuario puede obtener más detalles sobre la regulación y características geográficas. Las consultas espaciales las define el usuario en función del espacio geográfico. Se entiende por espacio geográfico aquel punto -par de coor- 
denadas geográficas-, círculo -radio-, itinerario -polilínea-, polígono, o un ámbito territorial a los que se suele disponer normativa a aplicar sea el caso de la parcela catastral o el límite administrativo municipal.



Figura 3. Consulta del RJT del Galacho de Juslibol. Zaragoza. Limitaciones ambientales. Fuente: IDEARAGON.

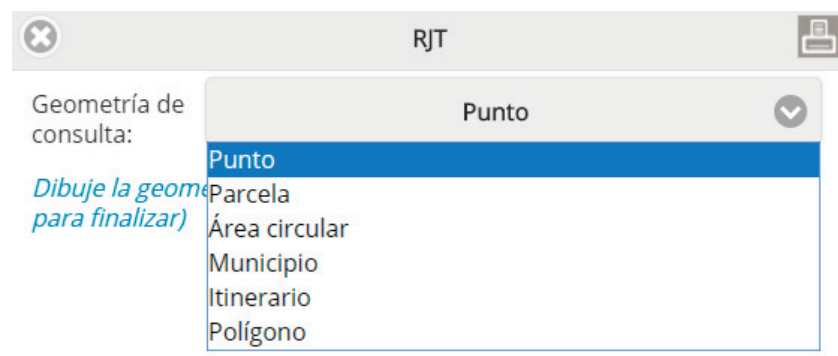

Dibuje la geometría sobre el mapa (para líneas y polígonos, doble click para finalizar)

\begin{tabular}{l|l|}
\hline $\begin{array}{l}\text { Tipo de } \\
\text { consulta: }\end{array}$ & \multicolumn{1}{c|}{ Ordenación del Territorio / Urbanismo } \\
\hline & \begin{tabular}{l} 
Ordenación del Territorio / Urbanismo \\
\hline $\begin{array}{l}\text { Ambiental } \\
\text { Sectorial }\end{array}$ \\
\hline
\end{tabular} \\
\hline
\end{tabular}

Figura 4. Cuadro de diálogo de selección de geometría y tipo de consulta del RJT.

Fuente: IDEARAGON. 
La información legal se divide en dos grupos: general (común a todas las características de un tema) y específico (sólo para la zona espacial consultada). También proporciona documentos complementarios o enlaces a sitios web externos que pueden ser útiles. Los datos recopilados y gestionados por esta herramienta ${ }^{2}$ son: 224 referencias generales a la normativa (ley, decretos, orden...), 103 referencias específicas a las regulaciones y 40 capas de información geográfica integradas. A la hora de seleccionar la información legal, hay que alcanzar un compromiso entre el nivel de detalle a mostrar y evitar la sobrecarga excesiva de información. Por ello, se ha aplicado el criterio de seleccionar exclusivamente la normativa más específica y representativa del tema que se considere. Se evita incorporar normas más generales y conocidas - léase por ejemplo la Constitución Española- pese a que sean también de aplicación en el territorio. De entre el articulado de esa normativa seleccionada, en lo posible se han incluido artículos descriptivos -más estables en el tiempo- y no los interpretativos, susceptibles de ser modificados posteriormente con una mayor probabilidad.

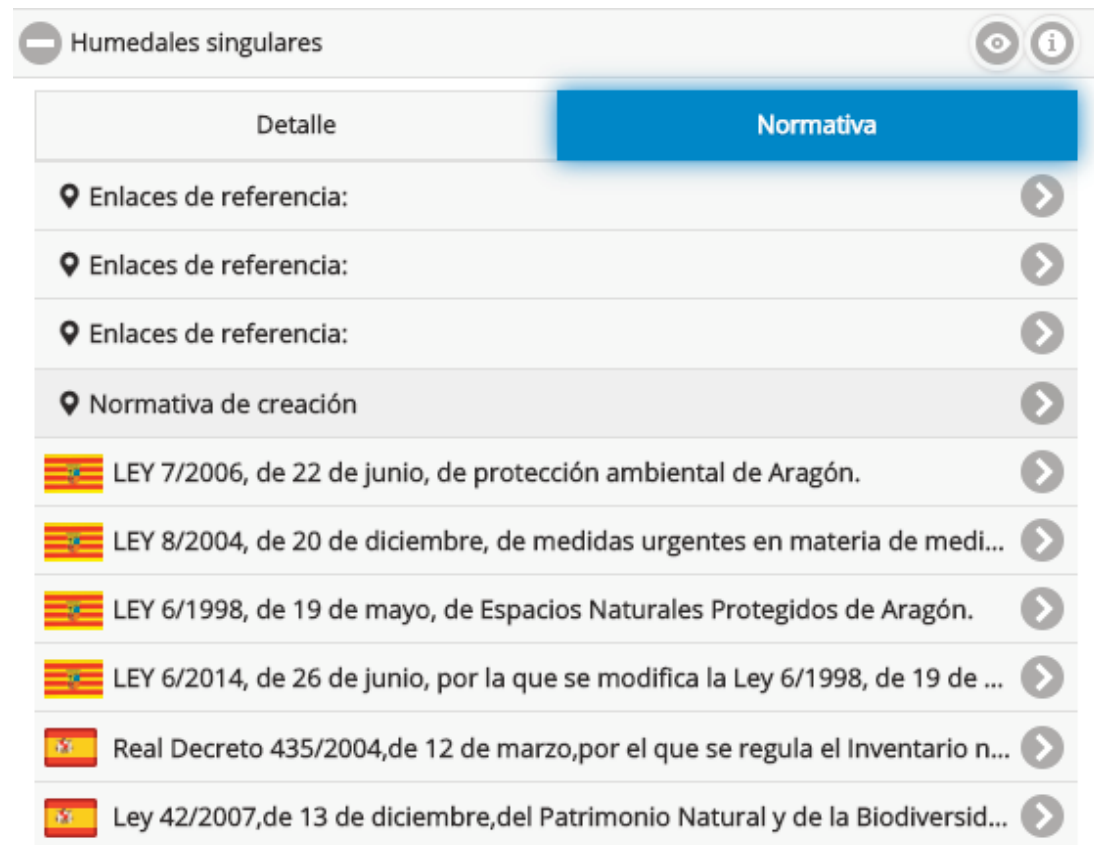

Figura 5. Detalle de la normativa general y específica de la figura de protección ambiental. Fuente: IDEARAGON.

2. Última consulta a la tabla de normativa del RJT realizada el 27 de Febrero de 2020. 
Históricamente, el proceso de gestión y recopilación de legislación en IDEARAGON ha sido manual, lo que requiere una carga de trabajo intensa para su actualización. Es decir, "se proporciona información legal para el consumo humano, pero la información generalmente no está disponible como datos para el análisis algorítmico y las aplicaciones a utilizar" (Oksanen et al., 2018). Para cada elemento, se ha almacenado la siguiente información: título, alcance (europeo, nacional, regional o mundial), tema, tipo (general o específico), fechas (inicio y fin), URL al Diario Oficial, identificador (para restricciones específicas) e información adicional.

"Las fuentes legales crecen en número y complejidad, al igual que la necesidad de informarse sobre ellas" (Winkels et al., 2002). Abordar este amplio conjunto de recursos jurídicos es un desafío para los responsables de la toma de decisiones, por lo que tener una forma sencilla de obtener una legislación consolidada es un objetivo principal. Durante los últimos años, se ha planteado una nueva iniciativa para ayudar en esta tarea denominada European Legislation Identifier (ELI). Dicha iniciativa "tiene como objetivo introducir la legislación en la Web global de datos, para facilitar el acceso, el intercambio y la interconexión de información jurídica" (Francart et al., 2019), hace que la legislación esté disponible en línea en un formato normalizado y está previsto que esté disponible para todos los niveles administrativos involucrados (europeo, nacional, regional y local). Se ha definido una ontología y, en este momento, hay datos vinculados (en formato RDF) sobre la legislación española y europea disponibles mediante URI http (identificadores persistentes en el tiempo). En IDEARAGON, se ha ampliado la información anterior sobre un elemento de regulación para almacenar un URI HTTP de ELI. Al recopilar el URI ELI correspondiente para cada dato legislativo almacenado previamente en IDEARAGON, se ha logrado una mejora del proceso de gestión de la información. Los títulos en varios idiomas, fechas relacionadas, versiones, temas, valor legal, identificadores persistentes, URL de acceso al Diario Oficial, etc. se pueden obtener automáticamente accediendo a los datos vinculados publicados en RDF.

Como consecuencia, la mayor parte de la información sobre la regulación se puede obtener automáticamente, y este sistema solo debe almacenar sus propios campos específicos: tema, tipo y características implicadas (si es específico), aparte del identificador URI ELI. De esta manera, el esfuerzo humano se ha reducido y la posibilidad de errores tipográficos es mínima. El acceso a versiones consolidadas de objetos legales se alcanza sin esfuerzo adicional, por lo que es una gran ventaja para los responsables de la toma de decisiones, especialmente cuando el marco legal es extenso.

\section{Resultados}

El resultado operativo es consultable a través de la siguiente dirección web: https:// idearagon.aragon.es/visor. En la aplicación web geográfica visor 2D de IDEARAGON está implementada la herramienta del RJT. 
Los gestores públicos que utilizan de forma regular la herramienta son los siguientes; para la gestión de procedimientos administrativos vinculados a organismos colegiados: Consejo de Ordenación del Territorio de Aragón (COTA), Consejo de Urbanismo de Aragón (CUA) y Consejos Provinciales de Urbanismo (CPU), y para la consulta, gestión de expedientes y toma de decisiones: Dirección General de Ordenación del Territorio, Dirección General de Urbanismo e Instituto Aragonés de Gestión Ambiental (INAGA). Se desconoce, de forma cuantitativa, el uso privado de la herramienta dado que el número de accesos / usos está integrado a nivel de aplicación en el visor 2D. IDEARAGON arroja una media de 40.000 de accesos mensuales al portal en el que se integran los servicios de consulta y visualización de información geográfica entre los cuales se incluye el RJT.

El desarrollo previsto, a corto plazo de la presente herramienta, es el siguiente; la gestión de histórico tanto de las referencias legales como de la información geográfica asociada a un determinado anuncio legal, la integración de datos de legislación vecina, la internacionalización de datos legislativos y la obtención de estadísticas específicas de acceso y uso a la presente herramienta tanto de uso interno (Intranet) como externo (Internet).

\section{Discusión}

El hándicap principal de esta herramienta radica en su carácter informativo. Tal como puede leerse en su cláusula de responsabilidad, esta herramienta no refleja el carácter de oficialidad que se le espera como a un boletín oficial. La realidad, uno, es que la distorsión entre la calidad de datos geográficos, y la enumeración de capas de información geográfica y conjunto de limitaciones y restricciones que se asocian, complican la veracidad del dato de respuesta de una herramienta como es el RJT. Y, dos, la falta de sincronización que existe aún entre lo exigido en la práctica legal y la información geográfica relacionada existente o no en la base de datos geográfica.

Este es el desafío a afrontar. Es decir, la aplicabilidad de servicios oficiales de visualización como prueba fehaciente en procesos judiciales y el concepto de interoperabilidad jurídica de los datos geográficos en el derecho del administrado. Hay que redoblar e intensificar esfuerzos en los dos prismas; el geográfico y el jurídico. Ambos han de estar ligados desde la propia redacción de las disposiciones legales. Ser coherentes en la redacción geojurídica, ayudará a que el tomador de decisiones pueda realizar procedimientos administrativos acordes con la realidad física y las limitaciones existentes en el territorio. La relevancia del dato geográfico, también en lo jurídico, está en que habla del dónde. "A diferencia de otras clases de datos, los datos espaciales tienen un elevado valor añadido geoestratégico. Estos pueden ser datos personales cuando identifiquen o permitan identificar a una persona física individual. En consecuen- 
cia, los derechos de los ciudadanos sobre los datos geoespaciales, cuando sean datos personales, son los derechos de protección de datos ya existentes" (Díaz, 2018).

El principal riesgo del dato geográfico es la calidad y la interoperabilidad. La falta de calidad y/o interoperabilidad de los datos geográficos constituye un riesgo para la efectiva prestación de servicios como el que realiza el RJT. Por lo que la aplicación de la Directiva del Parlamento Europeo y del Consejo del 14 de marzo de 2007, por la que se establece una infraestructura de información espacial en la Comunidad Europea (Inspire) es clave para el avance en este tipo de servicios que ofrece una regulación concreta sobre los datos y servicios geoespaciales, con particular atención a la interoperabilidad de los servicios en los Estados miembros.

La interoperabilidad jurídica de la geoinformación permite la relación e interacción entre los agentes jurídicos y el personal técnico implicados en actuaciones, procesos y procedimientos jurídicos ya sean administrativos, judiciales o extrajudiciales. Esta interoperabilidad jurídica contiene directas implicaciones sociales, económicas, administrativas y jurídicas. "El valor jurídico añadido de la geoinformación es, entre otros muchos, principalmente doble, como infraestructura y como servicio, pues allí donde hay espacio hay derecho" (Díaz, 2018).

\section{Conclusiones}

IGEAR suma un nuevo servicio web geográfico que ayuda a la toma de decisiones de agentes públicos o privados a través de la herramienta del RJT. Como organismo responsable de la información geográfica entiende que su labor no solo se circunscribe en la mera producción, almacenamiento y puesta a disposición de la información geográfica que se le confiere. El paso que está dando con esta herramienta y otros servicios y aplicaciones de su nodo IDE es más exigente. Requiere de un mayor esfuerzo cognitivo y técnico, pero el resultado es más provechoso dado que dota y acerca la información geográfica al ciudadano dando un mejor servicio al mismo. La ayuda en toma de decisiones para los actores de una IDE, se convierte en eje fundamental del catálogo de servicios del IGEAR. Y necesariamente, ese catálogo pasa por relacionar la información geográfica con los diferentes repositorios de información, como en este caso es el estrictamente jurídico. El apellido geográfico sucede en el territorio que se legisla. En suma, la herramienta del RJT es servicio de apoyo para que gentes públicos y privados se informen y adquieran evidencias geojurídicas necesarias para realizar una determinada actuación o acción legal sobre Aragón. 


\section{Bibliografía}

Abad, P. Bernabé, M.A. y Rodríguez, A. (2012). Compartir: la solución está en las Infraestructuras de Datos Espaciales. En Bernabé Poveda, M.A. y López Vázquez, C.M. (eds.) Fundamentos de las Infraestructuras de Datos Espaciales (pp. 41-53). Madrid: UPM Press.

Boer, A., Van Engers, T., Peters, R. and Winkels, R. (2007). Separating law from Geography in GISbased eGovernment services. Artif. Intell. Law, 15, pp. 49-76, 10.1007/s10506-007-9042-4. DOI: http://dx.doi.org/10.1007/s10506-007-9042-4

Departamento de asuntos regulatorios y europeos. CEOE (2019). La producción normativa en 2018. CEOE. https://contenidos.ceoe.es/CEOE/var/pool/pdf/publications_docs-file-601-laproduccion- normativa-en-2018.pdf.

Díaz, E. (2018). Uso legal de información geoespacial: Gestión jurídica, administrativa y judicial de responsabilidad patrimonial de la Administración pública por inundaciones. Editorial Académica Española.

Francart, T., Dann, J., Pappalardo, R., Malagon, C. and Pellegrino, M. (2019). The European Legislation Identifier. Knowledge of the Law in the Big Data Age, pp. 137-148. http://ebooks.iospress. $\mathrm{nl} /$ volumearticle/51782 (2019-12-26).

Oksanen, A., Tuominen, J.A., Mäkelä, E., Tamper, M., Hietanen, A. and Hyvönen, E.A. (2018). Semantic Finlex: Finnish Legislation and Case. Law as a Linked Open Data Service. Knowledge of the Law in the Big Data Age, pp. 212-228. https://seco.cs.aalto.fi/publications/2018/oksanen-et-al-lvi-2018.pdf

Portolés Rodríguez, D., Martínez, R. and López, F. (2019). Using ELI linked data for improving the information management in a legal regime visualization tool. Jornadas Ibéricas de Infraestructuras de Datos Espaciales 2019, 23-25 de octubre, Cáceres, España.

Sneiders, E. (2004). Tool support for legal enquiry. Focus on spatial-planning law. 6th International Conference on Enterprise Information Systems (ICEIS'O4), 14-17 de abril, Porto, Portugal.

Winkels, R., Boer, A. and Hoekstra, R. (2002). CLIME: Lessons Learned in Legal Information Serving. Phycologia, pp. 230-234. http://citeseerx.ist.psu.edu/viewdoc/download?doi=10.1.1.208.9744 \&rep=rep1\&type=pdf. 